\title{
Analysis of Fatty Acids in Virgin Coconut Oil Frying at Various Temperatures
}

\author{
Dewa Ayu Ika Pramitha ${ }^{1^{*}}$, I Wayan Karta ${ }^{2}$ \\ ${ }^{1}$ Program Studi DIII Farmasi, Fakultas Farmasi, Universitas Mahasaraswati \\ Denpasar, Indonesia \\ ${ }^{2}$ Teknologi Laboratorium Medis, Poltekkes Kemenkes Denpasar \\ Denpasar, Indonesia
}

e-mail: ika.pramitha20@gmail.com, iwayankartaganesh@gmail.com

\begin{abstract}
Fatty acid content from virgin coconut oil (VCO) is a medium-chain triglyceride (MCT) group. MCT is stable at very low and high temperatures, and the color does not turn black due to the addition of heat so that it can be developed into beneficial cooking oil for health. Therefore, a study was conducted on the content of fatty acids in VCO after being heated at temperatures of 150,200 , and $250^{\circ} \mathrm{C}$ for 60 minutes. Analysis of fatty acid content in control $\mathrm{VCO}$ (T0), $\mathrm{VCO}$ with heating temperatures of $150^{\circ} \mathrm{C}(\mathrm{T} 1), 200^{\circ} \mathrm{C}(\mathrm{T} 2)$, and $250^{\circ} \mathrm{C}$ (T3) was performed with GCMS QP-2010 Ultra.The results showed that there were differences in levels and types of fatty acids in VCO by treating T0, T1, T2, and T3. At these three temperatures still produce medium-chain saturated fatty acids and trans fatty acids are not produced, so that VCO can be utilized as cooking oil that has better stability and benefits for health.
\end{abstract}

Keywords : virgin coconut oil, frying, oils, fatty acids

\section{INTRODUCTION}

In general, cooking oil used in the frying process is coconut oil. There are three types of coconut oil, namely, processed coconut oil, copra oil, and virgin coconut oil (VCO). The fatty acid content of the three coconut oils is the same, but VCO (Virgin Coconut Oil) has a higher content of monoglycerides and diglycerides than other types of coconut oil and has other beneficial contents such as antioxidants (Dayrit, 2014). It has been reported that VCO can be a good cooking oil because it has relatively high oxidative stability compared to Extra Virgin Olive Oil on heating at $190^{\circ} \mathrm{C}$ for 40 days of storage. This is because VCO has more saturated fatty acid content (Lu \& Tan, 2009). VCO is considered a saturated oil because it has about $92 \%$ saturated fatty acids starting from caproic and stearic acids, and only about $8 \%$ unsaturated fatty acids consisting of oleic and linoleic acids.

VCO damage can be caused by oxidation, hydrolysis, and microbial contamination which is indicated by a change in the physicochemical properties of
VCO itself. The most important conditions affecting the physicochemical degradation of VCO are humidity, temperature, and the presence of microorganisms. Temperature is a very important factor in evaluating the oxidation stability of fats, especially unsaturated fats, because the oxidation mechanism with temperature and hydroperoxides differ in linoleic acid, which acts as a volatile precursor, and decomposes at different temperatures. The oxidation rate is exponentially related to temperature. So there is an algorithmic decrease in the shelf life of food lipids. An increase in temperature during the frying process will increase oxidation and thermal oligomerization reactions, not only from fatty acid molecules or triacylglycerol but also small components that cannot be certified (Alzaa, 2018). This temperature effect will have an impact on the frying process.

During fat frying, various deteriorative chemical processes occur such as hydrolysis, oxidation, cyclization, and polymerization, then the oil decomposes to form volatile products and various non- 
volatile compounds. In the presence of continuous heating and frying, these compounds decompose further until an accumulation of product breakdown results in a bad taste and potentially toxic effects, making the oil unsuitable for frying (Sharoba \& Fawzy Ramadan, 2012). The number of chemical compounds and structures formed depends on various factors, including oil and food type, frying conditions, and oxygen availability. This process can also reduce the number of antioxidants in oil, reduce its stability and produce new products that are responsible for the loss of nutritional value and oil quality (odor, aroma, absorption, etc.). Oxidized fatty acid products provide favorites and odors (hydrolytic rancidity) for medium foods and fried foods. Hydrolysis increases the amount of free fatty acids, mono and diacylglycerol, and glycerol in oil. Oxidation occurs at a greater rate than hydrolysis during deep-fat frying. Oxidation produces hydroperoxides and then low molecular volatile compounds such as aldehydes, ketones, carboxylic acids, and short-chain alkanes and alkenes (Choe \& Min, 2007).

Chemical changes that occur in cooking oil also produce changes in the quality of fried foods. The composition of fatty acids from cooking oil is an important factor affecting the taste of fried food and its stability. Therefore, low levels of polyunsaturated fatty acids such as linoleic or linolenic acids and high levels of oleic acid with moderate amounts of saturated fatty acids. As a result, the quality of frying oil is important because of the absorbed oil of fried products during deep frying. The frying quality of oil with high unsaturated fatty acids and free fatty acids is not as good as oil with low unsaturated fatty acids and free fatty acids. In this study (Derlean, 2009) removing the smell and taste of coconut oil is done by heating coconut oil at temperatures of 80 , 100 , and $150^{\circ} \mathrm{C}$ in 1,2 , and 3 hours. The results obtained in the study indicate that the heating treatment on VCO does not affect the content of fatty acids but increases free fatty acids and peroxide values. The determination of free fatty acids and peroxide values is carried out to determine the level of oil rancidity chemically. Therefore it is necessary to research the quality and content of fatty acids at heating temperatures of 150,200 , and $250^{\circ} \mathrm{C}$ for 60 minutes on VCO (Virgin Coconut Oil) which has been produced by natural fermentation for 24 hours. Based on this, the purpose of this study was to determine the fatty acid content in VCO after being heated to temperatures of 150,200 , and $250^{\circ} \mathrm{C}$ for 60 minutes.

\section{METHOD}

The research design used in this study is purely experimental. This research was conducted at the Joint Laboratory of the Faculty of Mathematics and Natural Sciences, Udayana University, Bukit Jimbaran, and the Saraswati Pharmacy Academy Laboratory, Denpasar. The variable of this research is the independent variable that is the volume of virgin coconut oil (VCO), the dependent variable is the content of fatty acids, and the control variables namely temperature and heating time.

\section{Material}

The materials used in this study were methanol, Whatman GF/C filter paper diameter $47 \mathrm{~mm}$ (CAT No. 1822-047), helium gas, natural fermented VCO, naturally fermented VCO samples after being heated to $150^{\circ} \mathrm{C}, 200^{\circ} \mathrm{C}$, and $250^{\circ} \mathrm{C}$ for 60 minutes.

The instruments used in this study were $0.1 \mu \mathrm{L}$ - $10 \mu \mathrm{L}$ micropipette (Raininpipette-one), $5 \mathrm{~mL}$ test tube (Iwaki), $10 \mu \mathrm{L}$ Syringe Microliter, Shimadzu GCMS QP2010 Ultra with MS detector under operating conditions: Trx-Restek column 5MS GC Columns with a length of $30 \mathrm{~m}$, diameter $0.25 \mathrm{~mm}$, film thickness $0.25 \mu \mathrm{m}$. The column temperature from $80^{\circ} \mathrm{C}$ for 2 minutes then increased to $270^{\circ} \mathrm{C}$ with the course of $10^{\circ} \mathrm{C} /$ minute and for 10 minutes the constant temperature was $270^{\circ} \mathrm{C}$. detector temperature $250^{\circ} \mathrm{C}$, injection temperature $250^{\circ} \mathrm{C}$ with helium carrier gas, gas flow rate $3.0 \mathrm{~mL} / \mathrm{min}$ and gas pressure $79.5 \mathrm{kPa}$.

\section{Procedure}

Prepared natural fermented VCO and natural fermented VCO which has been 
heated to a temperature of $150^{\circ} \mathrm{C}, 200^{\circ} \mathrm{C}$, and $250^{\circ} \mathrm{C}$ for 60 minutes each as much as $20 \mu \mathrm{L}$. The sample was dissolved with methanol in a $5 \mathrm{~mL}$ volumetric flask to the limit mark. Shake until homogeneous and filtered using Whatman GF/C filter paper. Each sample was taken as much as $1 \mu \mathrm{L}$ to be analyzed on the GCMS QP-2010 Ultra tool.

\section{RESULTS AND DISCUSSION}

The results of the analysis of fatty acid content in VCO heated at 150,200, and $250^{\circ} \mathrm{C}$ with GCMS as in Figure 1. Based on the GC-MS results it can be obtained the fatty acid content of each treatment as Table 1. The results of GC-MS analysis (Figure 1) show that in the VCO without heating there were six peaks which consisted of capric acid, lauric acid, myristic acid, palmitic acid, oleic acid, and tricapriline acid. In VCO heated at a temperature of $150^{\circ} \mathrm{C}(\mathrm{T} 1)$, there are 5 peaks where 2 peaks are having the same retention time so that in this VCO there are 4 fatty acids namely caprylic acid, capric acid, and palmitic acid. VCO heated at $200^{\circ} \mathrm{C}$ (T2) contains 4 types of compounds namely caprylic acid, capric acid, lauric acid, and palmitic acid. VCO heated at a temperature of $250^{\circ} \mathrm{C}$ contains 5 types of compounds namely caprylic acid, capric acid, lauric acid, myristic acid, palmitic acid.

Table 1 shows the variations in the content of the types of fatty acids found in each treatment. At the highest T0 content is myristic acid, then lauric acid, palmitic acid, and tricapriline acid, capric acid, and oleic acid. At a temperature of $150^{\circ} \mathrm{C}$, there are differences in the types of compounds and their levels, namely the presence of caprylic acid compounds $27.5 \%$, an increase in capric acid levels to $33.5 \%$, a decrease in lauric acid levels to $17.7 \%$, and an increase in levels of palmitic acid to $21.2 \%$. Heating at $200^{\circ} \mathrm{C}$ gives the results of the same type of fatty acids with $\mathrm{T} 1$, but the levels are different. The levels of caprylic acid, capric acid, and lauric acid at T2 are lower than those at $\mathrm{T} 1$, whereas palmitic acid has increased. Heating at $250^{\circ} \mathrm{C}$ provides different fatty acid content with T0, T1, and T2. At this temperature, there is a myristic acid whose level is lower than T0. The levels of caprylic acid are lower than those of $\mathrm{T} 1$ and T2. Capric acid levels are also lower compared to T1 and T2, but higher than T0. Lauric acid levels at T3 have higher levels than those at T1 and T2 but are still lower compared to T0. T3 contains myristic acid as in T0. Palmitic acid from T0 to T2 has increased, but at T3 has decreased.

Virgin coconut oil (VCO) is the purest type of coconut oil. The virgin coconut oil (VCO), which has been manufactured through fermentation and centrifugation technique has a lot of beneficial effects since it contains vitamin $\mathrm{E}$ and polyphenol10. Virgin coconut oil (VCO) is well known as a functional food that provides health and nutritional benefits. It provides over and beyond basic nutrients. VCO has been recognized in health food markets and has high dramatic growth in the international market. The emerging application of VCO in the health area strongly influenced by medium-chain fatty acids (MCFA) content such as lauric, myristic, palmitic, capric, stearic, oleic, and linoleic acids which are easily digestible. For instance, lauric acid, a medium-chain fatty acid component in VCO becomes potential use as anti-obesity treatment as it increases energy expenditure, directly absorbed and burnt as energy in the liver. As results thus leading to weight loss. Furthermore, VCO has multipurpose nutrient supplements such as vitamins, amino acids, antioxidants, antimicrobial, and antiviral compounds (Asiah et al., 2013)

Based on the standards of the APCC quality standard virgin coconut oil, the VCO produced in this study contains the type of fatty acids that conform to the standards, even though the levels are not too similar. In the process of heating at temperatures that have been determined, there is a degradation of natural types of fats and their contents. The initial VCO used in the study contained the origin of medium-chain saturated fats namely capric acid and lauric acid. In the long-chain saturated fatty acids contain meristic acid, palmitic acid. Unsaturated fatty acids contain oleic acid. On heating with various temperatures indicate various types of fatty acids. In frying, the total oxidation value and polar compound 
in VCO are lower than in palm olein due to the presence of polyphenol compounds (Omar et al., 2014). The process of frying by deep-frying, in addition to causing the formation of long-chain saturated fatty acids (LCFA), also causes thermal polymerization reactions and oxidation reactions that form trans fatty acids (TFA). The process of frying by deep-frying will cause the conversion of cis unsaturated fatty acids to trans form, and increase the number of trans fatty acids in proportion to the decrease in cis unsaturated fatty acids (oleic acid) (Sartika, 2008). The results of the GC-MS analysis showed that VCO with T1, T2, T3 treatments did not have trans fatty acids. The formation of TFA during food frying is closely related to the process temperature and oil use time (Martin et al., 2007).

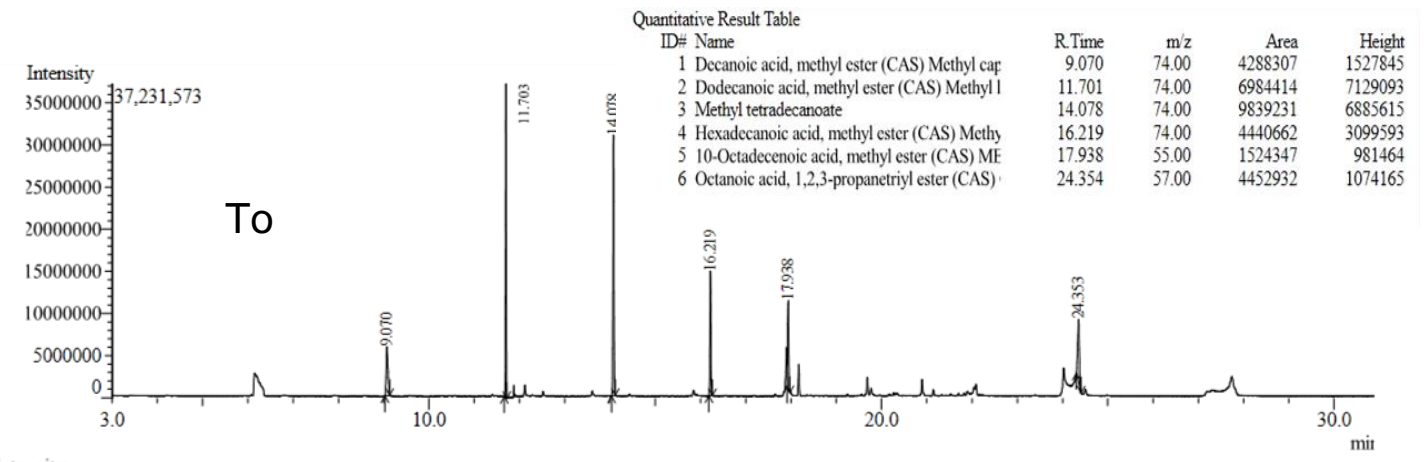

Intensity
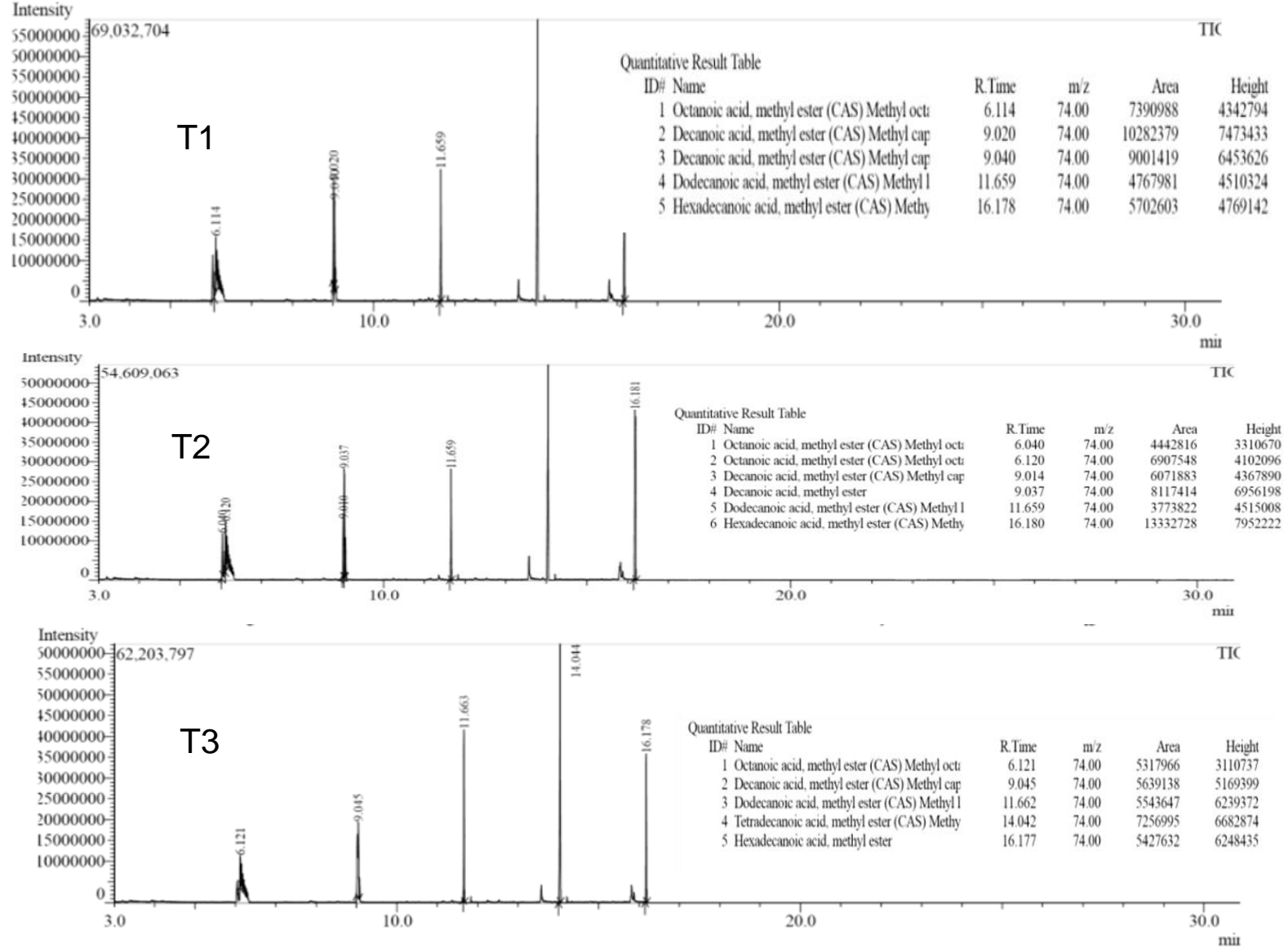

Figure 1. GC-MS Analysis Results of Methyl Esters on VCO treatment without frying (T0), Frying at a temperature of $150^{\circ} \mathrm{C}(\mathrm{T} 1), 200^{\circ} \mathrm{C}(\mathrm{T} 2)$, and $250^{\circ} \mathrm{C}(\mathrm{T} 3)$. 
Table 1. The content of fatty acids in each treatment

\begin{tabular}{lcccc}
\hline \multirow{2}{*}{\multicolumn{1}{c}{ Fatty acids }} & \multicolumn{5}{c}{ Content $(\%)$} \\
\cline { 2 - 5 } & T0 & T1 & T2 & T3 \\
\hline Caprylic acid & - & 27,5 & 21,5 & 18,2 \\
Capric acid & 13,6 & 33,5 & 25,3 & 19,3 \\
Lauric acid & 22,2 & 17,7 & 11,7 & 19,0 \\
Myristic acid & 31,2 & - & - & 24,9 \\
Palmitic acid & 14,1 & 21,2 & 41,5 & 18,6 \\
Oleic acid & 4,8 & - & - & - \\
Tricapriline acid & 14,1 & - & - & - \\
\hline
\end{tabular}

Trans fatty acids occur in food either naturally or produced during the heat processing of food containing unsaturated fats. Naturally occurring trans fatty acids have different physiological and biological functions as compared to those formed in heat-processed food which increases the risk of coronary heart disease (Afaneh et al., 2017). The significant increases in trans fatty acids in edible oils during frying and heating process. The higher temperature accelerates the reaction rates of oxidation and the more trans fat is generated (Song et al., 2015). Based on the results of the study showed that trans fatty acids are not produced, so potentially VCO can be used as cooking oil.

Oil damage due to the frying process at high temperatures $\left(200-250^{\circ} \mathrm{C}\right)$ that damage the double bonds in unsaturated fatty acids so that only fatty acids are left. Generally, oxidation damage occurs in unsaturated fatty acids, but if the oil is heated at a temperature of $100^{\circ} \mathrm{C}$ or more, saturated fatty acids can also be oxidized. The oxidation reaction at a frying temperature of $200^{\circ} \mathrm{C}$ makes it easier to damage oil with a high degree of unsaturation, while the hydrolysis reaction easily occurs in oils with long-chain saturated fatty acids (LCFA). The good heating temperature is around 95$120^{\circ} \mathrm{C}$. This can be seen from the results of research showing that at $\mathrm{T} 1, \mathrm{~T} 2$, and $\mathrm{T} 3$ there is no unsaturated chain. Lauric acid, which is a medium-chain saturated fatty acid at T1, T2, and T3, has decreased levels compared to levels at T0. This indicates the oxidation reaction that occurs when heating. All essential fatty acids are easily damaged by oxidation and heating reactions (Sartika, 2008). The oxidation reaction will encourage the formation of peroxide and hydroperoxide. Besides that, the higher the heating temperature, the faster the absorption of $\mathrm{O}_{2}$ into the unsaturated fatty acid radicals, the greater the consequence of peroxide compounds. The speed of the peroxide formed also depends on the composition of the oil. The oil that has a small unsaturated fatty acid content will be resistant to rancidity while oils that have a high unsaturated fatty acid content will easily undergo an oxidation process (Brühl, 2014). At high temperatures, linoleic acid can polymerize and form short-chain fatty acids. Oil damage after the deep frying process depends on the type of oil, the quality of fresh cooking oil, and the treatment of repetitive oils (Bazina \& $\mathrm{He}, 2018$ ). Based on the overall data of this study, it shows that VCO can still maintain levels of mediumchain saturated fatty acids in the temperature treatment, even though there is a decrease in levels.

At a heating temperature of $150^{\circ} \mathrm{C}$, it shows that the fatty acid content is better than at $200^{\circ} \mathrm{C}$ and $250^{\circ} \mathrm{C}$. This is because it still contains a lot of medium-chain saturated fatty acids and little long-chain saturated fatty acids. At T2, the abundance of longchain saturated fatty acids is higher, namely palmitic acid with $41.5 \%$ content. At T3, there appear two types of long-chain saturated fatty acids namely myristic acid and palmitic acid. VCO contains saturated fatty acids with medium chains. Saturated fatty acids withstand high temperatures when used in frying and are not easy to form trans fatty acids (TFA) so they can be used as safe food oils. High contents of TFA in foods are of concern for human health because they promote coronary heart disease. An 
increase in the blood levels of LDL (LDL) cholesterol and TAGs and a decrease in the level of HDL (HDL) cholesterol can be observed. There is further evidence for effects in connection with sudden cardiac death and systemic inflammation (Mozaffarian et al., 2009). VCO contains many types of fatty acids in the mediumchain triglyceride (MCT) group so that it has high oxidative stability. This oxidative stability is supported by the value of the active oxygen method (AOM) on MCT greater than other oils (Sahin, 2019). MCT is very stable at very low and high temperatures, and the color does not turn black due to the addition of heat. Pure coconut oil (VCO) can improve the performance of palm-based solid shortening fryers during deep frying. The physicochemical properties of the VCO: PS mixture is better than palm shortening (PS) alone. Also, in the case of sensory evaluation, the addition of virgin coconut oil to a palm-based solid shortening showed significant differences in the oiliness, taste, and overall acceptance of fried chicken after repeated frying. Overall, the addition of a small portion of VCO to shortening oil-based solids provides a large increase in the stability of oil frying (Omar et al., 2014). Therefore VCO can withstand heat temperatures and is also good for health. Based on the data in Table 1, VCO has a stability of heating up to $250^{\circ} \mathrm{C}$ and this can provide health benefits in its use for health.

Oil damage or degradation of fatty acid changes can be caused by heating or temperature, oxidation, and hydrolysis (Brühl, 2014). In the process of heating, the oil is damaged usually when used in frying, so the selection of the quality of cooking oil is needed. But the selection of cooking oil is not an easy task. The ideal oil fulfills all parameters from operational performance to quality and stability to nutrition. The physical and chemical properties of oil that are suitable for frying applications are very important in the selection of cooking oil. Melting point, heat content and heat capacity, and smoke point are critical properties of cooking oil. In the industry, the stability of cooking oil is also important. The stability of cooking oil was previously achieved mainly through the use of more saturated products, both naturally and by hydrogenation. However, hydrogenation produces trans fats, which (rightly or wrongly) are associated with an increased risk of heart disease. Consumers are now very aware that health is very important, and realize that food is not only pathologically safe (will not contribute to long-term or degenerative diseases) but are also physiologically healthy (has high nutritional qualities to support health). To meet the expectations and demands of consumers, the food industry around the world is reformulating a healthier diet, leaving behind traditional saturated fats and hydrogenated oils for healthier monounsaturated and polyunsaturated oils (Tian, 2013).

The frying process is a complex process that causes many physical and chemical changes to occur simultaneously in hot oil and fried foods in it. The quality of oil as a frying media is closely related to the quality of food produced in it (Brühl, 2014). High heat and long heating time increase the hydrolysis reaction to the negative secondary reaction become dominant. A decrease in oil quality results in a decrease in food characteristics such as darkening and surface hardening and excessive oil absorption, bad taste, and odor. There are three main types of reactions that occur during frying, namely hydrolysis, oxidation, and polymerization. Hydrolysis reaction to ester bonds occurs because of the moisture exhibited by food by releasing long-chain free fatty acids. Oxidation reactions can occur in the presence of water. While the polymerization reaction occurs due to radical recombination by forming -C-C-, -C-O-C-, and $-\mathrm{C}-\mathrm{O}-\mathrm{O}-\mathrm{C}-$ bonds. These three reactions are at the core of the current frying theory. High temperature has a very important role in the occurrence of polymerization reactions. Polymerization occurs in three ways: radical recombination, radical addition to the double bond, and the Diels-Alder reaction, the reaction between the double bond and conjugated diene (Tian, 2013).

Sufficient energy at high temperatures is used to break almost all types of bonds in the acyl or glycerol chains. Therefore, thermal degradation must be expected to 
form significantly different products from autoxidation products. the first process depends only on heat and not on oxygen, so as long as heat is used, degradation continues. This results in a large radical charge reacting with antioxidants and limiting its effectiveness in stabilizing cooking oil. Second, in contrast to oxidation, a series of homologous hydrocarbons are formed by cleavage at various positions in the acyl chain, then abstraction by alkyl radicals or by recombination of short-chain fragments. Dimers and polymers are also produced by radical recombination. Third, the presence of a radical separator adds oxygen to the terminal form of the peroxyl radical, which can selectively extract hydrogen from the allylic site to start the lipid oxidation chain reaction. At the same time, hydrogen abstraction produces terminal hydroperoxides which break down into oxygenated products with a long series of homologous chains (Tian, 2013). This is the cause of differences in the content and levels of fatty acids in each treatment T0, T1, T2, and T3. Many studies support that oils that contain polyunsaturated fatty acids must have lower frying stability than oils that contain monounsaturated and saturated fatty acids. Various oxidation products accumulate during long frying including free fatty acids, polar compounds, dimers, and polymers, at most at high levels. The effect of frying time becomes more significant in used oils that already have some degradation products and surfactants. High temperatures also accelerate oil degradation and affect the quality of fried foods. Sharoba and Ramadhan's research (2017) states that when the temperature of partially hydrogenated soybean oil frying increases from $160^{\circ} \mathrm{C}$ to $200^{\circ} \mathrm{C}$, acid and polymerization values increase, and the color of the oil becomes darker. The intermittent frying process generally causes more serious degradation than continuous frying. This is due to an increase in dissolving oxygen in the oil during the cooling period. For example, $25 \%$ of linoleic acid is degraded during intermittent frying compared to $5 \%$ during continuous frying (Tarmizi et al., 2016).

\section{CONCLUSION}

Based on the results of the GC-MS shows that there are differences in the content of fatty acids in VCO by treating T0, T1, T2, and T3. T0 treatments contained capric acid, lauric acid, myristic acid, palmitic acid, oleic acid, and tricapriline acid. At T1 and T2 there are caprylic acid, capric acid, lauric acid, and palmitic acid. The highest fatty acid level at $\mathrm{T} 1$ is capric acid $33.5 \%$ which is a medium-chain saturated fat, and there is a long chain saturated fat of palmitic fatty acid of $21.2 \%$. The highest fatty acid at T2 is $41.5 \%$ palmitic acid. At T3 there are caprylic acid, capric acid, lauric acid, myristic acid, palmitic acid, and the highest fatty acid is myristic acid $24.9 \%$. Fatty acid levels in each treatment have differences. At these three temperatures still produce mediumchain saturated fatty acids and trans fatty acids are not produced, so that VCO can be utilized as cooking oil that has better stability and benefits for health. Further research can be carried out on the analysis of fatty acid content with repeated frying.

\section{UCAPAN TERIMAKASIH}

Thank you conveyed to the Mahasaraswati University and also Pharmacy Faculty of Mahasaraswati University for supporting this study.

\section{DAFTAR PUSTAKA}

Afaneh, I., Abbadi, J., Al-Rimawi, F., AlDabbas, G., \& Sawalha, S. 2017. Effect of Frying Temperature and duration on the Formation of Trans Fatty Acids in Selected Fats and Oils. American Journal of Food Science and Technology, 5(6), 245-248.

https://doi.org/10.12691/ajfst-5-6-4

Alzaa, D. F. 2018. Evaluation of Chemical and Physical Changes in Different Commercial Oils during Heating. 2(6), 02-11.

Asiah, N., Cempaka, L., \& Maulidini, T. 2013. Comparative Study: PhysicoChemical Properties of Virgin Coconut Oil Using Various Culture. 6(2), 1-5. 
Bazina, N., \& He, J. 2018. Analysis of fatty acid profiles of free fatty acids generated in deep-frying process. Journal of Food Science and Technology, 55(8), 3085-3092. https://doi.org/10.1007/s13197-0183232-9

Brühl, L. 2014. Fatty acid alterations in oils and fats during heating and frying. European Journal of Lipid Science and Technology, 116(6), 707-715. https://doi.org/10.1002/ejlt.201300273

Choe, E., \& Min, D. B. 2007. Chemistry of Deep-Fat Frying Oils. Journal of Food Science, 72(5), R77-R86. https://doi.org/10.1111/j.17503841.2007.00352.x

Dayrit, F. M. 2014. Lauric Acid is a MediumChain Fatty Acid, Coconut Oil is a Medium-Chain Triglyceride. In Philippine Journal of Science (Vol. 143, Issue 2).

Lu, H., \& Tan, F. S. 2009. A Comparative study of storage stability in virgin coconut oil and extra virgin Olive oil upon thermal treatment. In International Food Research Journal (Vol. 16).

Martin, C. A., Milinsk, M. C., Visentainer, J. V, Matsushita, M., \& De-Souza, N. E. 2007. Trans fatty acid-forming processes in foods: a review. 79(2), 343-350. www.scielo.br/aabc

Mozaffarian, D., Aro, A., \& Willett, W. C. 2009. Health effects of trans-fatty acids: Experimental and observational evidence. European Journal of Clinical Nutrition, 63, S5-S21. https://doi.org/10.1038/sj.ejcn.1602973 Omar, M. N., Nor-Hazwani, M. H., Nazreen, M. N. M., \& Zuberdi, A. M. 2014. Studies on frying quality of virgin coconut oil and shortening blends.

Oriental Journal of Chemistry, 30(3), 1279-1286.

https://doi.org/10.13005/ojc/300344

Sahin, S. 2019. Evaluation of Stability against Oxidation in Edible Fats and Oils. Journal of Food Science and Nutrition Research, 02(03), 283-298. https://doi.org/10.26502/jfsnr.264211000027

Sartika, R. A. D. 2008. Pengaruh Asam Lemak Jenuh, Tidak Jenuh dan Asam Lemak Trans terhadap Kesehatan. Kesmas: National Public Health Journal, 2(4), 154. https://doi.org/10.21109/kesmas.v2i4.2 58

Sharoba, A. M., \& Fawzy Ramadan, M. 2012. Impact of Frying on Fatty Acid Profile and Rheological Behaviour of Some. https://doi.org/10.4172/21577110.1000161

Song, J., Park, J., Jung, J., Lee, C., Gim, S. Y., Ka, H., Yi, B., Kim, M.-J., Kim, C.-I., \& Lee, J. 2015. Analysis of Trans Fat in Edible Oils with Cooking Process. Toxicol. Res, 31(3), 307-312. https://doi.org/10.5487/TR.2015.31.3.3 07

Tarmizi, A. H. A., Ismail, R., \& Kuntom, A. 2016. Effect of frying on the palm oil quality attributes-A review. In Journal of Oil Palm Research (Vol. 28, Issue 2, pp. 143-153). Lembaga Minyak Sawit Malaysia. https://doi.org/10.21894/jopr.2016.280 2.01

Tian, X. 2013. Chemical Factors Affecting Degradation Processes Of Vegetable Oils During Frying. https://doi.org/https://doi.org/doi:10.728 2/T39S1P2C 